\title{
Nonlinear Schrödinger equation for a superfluid Bose gas from weak coupling to unitarity: Study of vortices
}

\author{
S. K. Adhikari ${ }^{1, *}$ and L. Salasnich ${ }^{2, \dagger}$ \\ ${ }^{1}$ Instituto de Física Teórica, UNESP_São Paulo State University, 01.405-900 São Paulo, São Paulo, Brazil \\ ${ }^{2}$ CNR-INFM and CNISM, Unità di Padova, Dipartimento di Fisica “Galileo Galilei,” Università di Padova, Via Marzolo 8, 35131 \\ Padova, Italy
}

(Received 17 December 2007; revised manuscript received 22 January 2008; published 18 March 2008)

\begin{abstract}
We introduce a nonlinear Schrödinger equation to describe the dynamics of a superfluid Bose gas in the crossover from the weak-coupling regime, where $a n^{1 / 3} \ll 1$ with $a$ the interatomic $s$-wave scattering length and $n$ the bosonic density, to the unitarity limit, where $a \rightarrow+\infty$. We call this equation the unitarity Schrödinger equation (USE). The zero-temperature bulk equation of state of this USE is parametrized by the Lee-YangHuang low-density expansion and Jastrow calculations at unitarity. With the help of the USE we study the profiles of quantized vortices and vortex-core radius in a uniform Bose gas. We also consider quantized vortices in a Bose gas under cylindrically symmetric harmonic confinement and study their profile and chemical potential using the USE and compare the results with those obtained from the Gross-Pitaevskii-type equations valid in the weak-coupling limit. Finally, the USE is applied to calculate the breathing modes of the confined Bose gas as a function of the scattering length.
\end{abstract}

DOI: 10.1103/PhysRevA.77.033618

PACS number(s): 03.75.Lm, 03.75.Kk, 03.75.Hh

\section{INTRODUCTION}

For the theoretical investigation of the Bose-Einstein condensate (BEC) of an ultracold bosonic gas the main tool is the mean-field Gross-Pitaevskii equation (GPE) [1,2], that is reliable for small values of the gas parameter $a n^{1 / 3}$, where $a$ is the $s$-wave scattering length of the interatomic potential and $n$ is the bosonic density. By manipulating a background magnetic field near a Feshbach resonance the $s$-wave scattering length $a$ can be modified and can reach very large values corresponding to a strong atomic interaction [3]. To take into account the effect of a large scattering length, a modified GPE (MGPE) has been introduced by Fabrocini and Polls [4] by using the first two terms in the Lee-Yang-Huang expansion [5] of the energy of a uniform Bose gas, which includes terms of the order of $\sqrt{n a^{3}}$. Within the density functional approach of Fabrocini and Polls [4], the leading term of expansion gives the GPE while the next term is responsible for corrections due to moderate atomic interaction. These two leading terms have been used to calculate beyond-mean-field corrections to properties of trapped BECs [6]. The contribution terms of the order of $n a^{3}$ has also been discussed in the literature [7].

In this paper we generalize the MGPE [4] by also considering the behavior of the bosonic system in the unitarity limit, where $a \rightarrow+\infty$. Jastrow calculations [8,9] suggest that in the unitarity limit the zero-temperature bulk chemical potential $\mu$ of the Bose system is given by $\mu=\xi n^{2 / 3} \hbar^{2} / m$, where $\xi$ is a constant and $m$ is the mass of a single atom. (Bulk chemical potential is essentially the nonlinear term that appears in the mean-field equation and is related to the energy per particle of the system.) This result is independent of the atomic scattering length. In the present work the equa-

\footnotetext{
*adhikari@ift.unesp.br; URL: www.ift.unesp.br/users/adhikari

†salasnich@pd.infn.it; URL: www.padova.infm.it/salasnich
}

tion of state of the bulk system is parametrized with a Padè approximant by using the first two terms of the Lee-YangHuang expansion [5] and the Jastrow calculations at unitarity $[8,9]$. In this way we obtain a time-dependent highly nonlinear Schrödinger equation that we call the unitarity Schrödinger equation (USE). The USE in general form is time dependent and can be used to study nonstationary dynamics, whereas its time-independent form is appropriate to study stationary states. The USE gives the hydrodynamic equations of bosonic superfluids at zero temperature and enables one to study collective dynamical properties of the system in the full crossover from weak-coupling to unitarity.

As an application of the USE, here we study the structure of quantized vortices in both uniform Bose gas and Bose gas under axially symmetric harmonic confinement and compare and contrast the results with those obtained with the MGPE [4] and GPE. Quantized vortices in superfluids are a manifestation of quantum mechanics at the macroscopic level [10]. Recently quantized vortices have been observed in rotating ultracold atomic BECs [11] and also in atomic Fermi gases in the BCS-BEC crossover near a Feshbach resonance [12]. Using the GPE, quantized vortices in a harmonic trap have been analyzed by Dalfovo and Stringari [13]. More recently vortices have been investigated with GPE in various problems; for instance, collective modes of a vortex [14], vortex under toroidal confinement $[15,16]$, vortex lattice [17], vortex with attractive scattering length $[18,19]$, collision dynamics of vortices [20], collapse of a vortex state [21], free expansion of vortices [22], and vortex in BoseFermi mixtures [23]. Nilsen et al. [24] used the MGPE [4] to investigate the structure of vortices and found good agreement between the MGPE and variational Monte Carlo results. Using the USE, we extend the study of Nilsen et al. [24] and find that the radius of the vortex core decreases with the increase of the scattering length. At the unitarity limit it reaches a critical minimal radius. The properties of this minimal radius depend on the trapping geometry: in the case of a 
vortex in a uniform Bose gas it is a decreasing function of the uniform density at large distances; in the case of a vortex in the harmonic trap it is a decreasing function of the total number of atoms. Using the USE, we also calculate the radial and axial frequencies of collective oscillations from weak-coupling to unitarity in a cigar-shaped trap.

In Sec. II we introduce the present model and relate it to superfluid hydrodynamics. Formulation for quantized vortices in the present model is considered in Sec. III. Section IV is devoted to a vortex in a uniform Bose gas, where we numerically study the vortex profile and vortex-core radius for different scattering lengths. In this case the USE is solved by the fourth-order Runge-Kutta method [25,26]. The vortex-core radius decreases with increasing scattering length and saturates to a constant value in the unitarity limit. In Sec. V we consider a vortex in a Bose gas under axially symmetric harmonic pancake-shaped confinement by solving the USE by imaginary time propagation using the semiimplicit Crank-Nicholson rule [26-28]. In this case we study the profiles of vortices and corresponding chemical potentials and compare the results with those obtained from the MGPE [4] and GPE. The interesting feature of the results of the USE is that they saturate in the unitarity limit as $a \rightarrow \infty$, whereas the results of MGPE and GPE do not saturate in this limit. The frequencies of collective breathing oscillations in a cigar-shaped trap are considered in Sec. VI. Finally, in Sec. VII we present the concluding remarks.

\section{SUPERFLUID HYDRODYNAMICS AND NONLINEAR SCHRÖDINGER EQUATION}

The zero-temperature collective properties of a dilute bosonic superfluid under the external potential $U(\mathbf{r})$ can be described by the quantum hydrodynamic equations $[29,30]$ :

$$
\begin{gathered}
\frac{\partial n}{\partial t}+\nabla \cdot(n \mathbf{v})=0 \\
m \frac{\partial \mathbf{v}}{\partial t}+\nabla\left[-\frac{\hbar^{2}}{2 m} \frac{\nabla^{2} \sqrt{n}}{\sqrt{n}}+\frac{m}{2} v^{2}+U+\mu(n, a)\right]=0,
\end{gathered}
$$

where $n(\mathbf{r}, t)$ is the local density and $\mathbf{v}(\mathbf{r}, t)$ is the local velocity of the bosonic system. In these zero-temperature hydrodynamical equations statistics enter the equation of state through the bulk chemical potential $\mu(n, a)$ and the quantumpressure term $-\left[\hbar^{2} /(2 m \sqrt{n})\right] \nabla^{2} \sqrt{n}$, which is absent in the classical hydrodynamic equations [31]. This hydrodynamic regime is achieved in the limit of a very large number of atoms $N$. At zero temperature, the bulk chemical potential $\mu(n, a)$ of the system is a function of the density $n$ and of the interatomic scattering length $a$. For a superfluid the velocity field is irrotational, i.e., $\boldsymbol{\nabla} \times \mathbf{v}=0$, and the circulation is quantized, i.e.,

$$
\oint \mathbf{v} \cdot d \mathbf{r}=2 \pi L \frac{\hbar}{m},
$$

where $L$ is an integer (angular momentum) quantum number [10].
We can introduce the complex order parameter $[2,32]$ $\Psi(\mathbf{r}, t)=n(\mathbf{r}, t)^{1 / 2} e^{i S(\mathbf{r}, t)}$ such that the phase $S(\mathbf{r}, t)$ of the order parameter fixes the superfluid velocity field

$$
n(\mathbf{r}, t) \mathbf{v}(\mathbf{r}, t)=-i \frac{\hbar}{2 m}\left(\Psi^{*} \nabla \Psi-\Psi \nabla \Psi^{*}\right)
$$

so that $\mathbf{v}(\mathbf{r}, t)=(\hbar / m) \nabla S(\mathbf{r}, t)$. In this way we can map Eqs. (1) and (2) into the following time-dependent highly nonlinear Schrödinger equation:

$$
i \hbar \frac{\partial}{\partial t} \Psi=\left[-\frac{\hbar^{2}}{2 m} \nabla^{2}+U(\mathbf{r})+\mu(n, a)\right] \Psi .
$$

In general, one can use the hydrodynamic equations (1) and (2), or equivalently Eq. (5), to study the global properties of the superfluid, like the stationary density profile, the free expansion, and the collective oscillations.

For a Bose gas the following two leading terms of the low-density expansion of the bulk chemical potential can be obtained [24] from the expression for energy per particle as obtained by Lee, Yang, and Huang [5]:

$$
\mu(n, a)=\frac{4 \pi \hbar^{2}}{m} \text { an }\left(1+\frac{32}{3 \pi^{1 / 2}}\left(n^{1 / 3} a\right)^{3 / 2}+\cdots\right),
$$

where $n^{1 / 3} a$ is the dimensionless gas parameter [32]. Note that in this expansion the scattering length $a$ must be positive $(a>0)$ corresponding to a repulsive interaction. Higher order correction terms to the bulk chemical potential have also been considered in the literature [7]. The lowest order term of expansion (6) was derived by Lenz [33]. Considering only this term, Eq. (5) becomes the familiar GPE $[1,2]$

$$
i \hbar \frac{\partial}{\partial t} \Psi=\left[-\frac{\hbar^{2}}{2 m} \nabla^{2}+U(\mathbf{r})+\frac{4 \pi \hbar^{2}}{m} a|\Psi|^{2}\right] \Psi .
$$

By also taking into account the second term of the expansion (6), Eq. (5) becomes the so-called MGPE introduced by Fabrocini and Polls [4]:

$$
\begin{aligned}
i \hbar \frac{\partial}{\partial t} \Psi= & {\left[-\frac{\hbar^{2}}{2 m} \nabla^{2}+U(\mathbf{r})\right.} \\
& \left.+\frac{4 \pi \hbar^{2}}{m} a|\Psi|^{2}\left(1+\frac{32}{3 \sqrt{\pi}} a^{3 / 2}|\Psi|\right)\right] \Psi .
\end{aligned}
$$

In the unitarity limit, where $a \rightarrow+\infty$, for dimensional reasons $[8,9]$ the bulk chemical potential must be of the form [8]

$$
\mu(n, a)=\xi \frac{\hbar^{2}}{m} n^{2 / 3},
$$

where $\xi$ is a universal coefficient. Thus in the unitarity limit the bulk chemical potential is proportional to that of a noninteracting Fermi gas [34]. Recent numerical calculations based on Jastrow variational wave functions give $\xi=22.22$ [8] and we shall consider this value of $\xi$ in our calculation.

In the full crossover from the small-gas-parameter regime to the large-gas-parameter regime, we suggest the following expression as the bulk chemical potential of the Bose superfluid: 


$$
\mu(n, a)=\frac{\hbar^{2}}{m} n^{2 / 3} f\left(n^{1 / 3} a\right),
$$

where $f(x)$ is an unknown dimensionless universal function of the gas parameter $x=n^{1 / 3} a$. A general Padè approximant for the function $f(x)$ consistent with the expansion of Lee, Huang, and Yang [5]Eqs. (6) and (10) should have the following form:

$$
f(x)=4 \pi \frac{x+\alpha x^{5 / 2}}{1+\gamma x^{3 / 2}+\beta x^{5 / 2}},
$$

where $\alpha, \beta$, and $\gamma$ are yet undetermined parameters. Without further information about $\mu(n, a)$ we cannot determine all these parameters consistently. In this paper we consider the minimal form of this function consistent with Eqs. (6) and (10) obtained by setting $\gamma=0$ and $\alpha=32 /(3 \sqrt{\pi})$ and $\beta$ $=4 \pi \alpha / \xi$, with $\xi=22.22$. The function $f(x)$ of Eq. (11) is such that $f(x)=4 \pi\left[x+32 x^{5 / 2} /(3 \sqrt{\pi})\right]$ for $x \ll 1$ and the present model reduces to the MGPE (8) [4]. In the opposite extreme $x \gg 1, f(x)=\xi$, and the present model reduces to the unitarity limit (9). We call Eq. (5) equipped with Eqs. (10) and (11), e.g.,

$$
\begin{aligned}
i \hbar \frac{\partial}{\partial t} \Psi= & {\left[-\frac{\hbar^{2}}{2 m} \nabla^{2}+U(\mathbf{r})\right.} \\
& \left.+\frac{4 \pi \hbar^{2}}{m} a|\Psi|^{2}\left(\frac{1+\alpha a^{3 / 2}|\Psi|}{1+\beta a^{5 / 2}|\Psi|^{5 / 3}}\right)\right] \Psi
\end{aligned}
$$

by the name USE, i.e., unitarity Schrödinger equation. As, by construction, the USE has the proper weak [24] and strong [8] coupling limits, it is appropriate for the study of weakto-strong coupling crossover. This is the model equation we use in the following sections to study quantized vortices in a Bose superfluid from weak to strong coupling. In this section $\Psi$ is normalized by $\int|\Psi|^{2} d \mathbf{r}=N$.

It is important to stress that the present approach, based on the quantum hydrodynamics, could be applied to both superfluid bosons and fermions $[29,35]$. This hydrodynamic approach is a time-dependent local density approximation with gradient corrections. In the last few years we have successfully applied it to investigate the collective properties of different dilute systems, like Bose-Fermi mixtures [36], the superfluid Fermi gas in the BCS-BEC crossover [37], and the one-dimensional (1D) Lieb-Liniger liquid [38]. Here we have obtained the USE valid from weak-coupling to unitarity based on the general quantum hydrodynamical scheme.

\section{QUANTIZED VORTICES}

The structure of vortices in superfluid ${ }^{4} \mathrm{He}$ at zero temperature was investigated many years ago by Chester, Metz, and Reatto [39] by using a many-body variational wave function, and more recently by Dalfovo [40] by using the Orsay-Trento density functional [41]. The two approaches, which give very similar results, are based on the assumption that the superfluid velocity of the quantized vortex rotating around the cylindric $z$ axis is given by

$$
\mathbf{v}=\frac{\hbar}{m} \frac{L}{\rho} \mathbf{u}_{\phi}
$$

where $L$ is the quantum number of circulation, $\rho$ is the cylindric radial coordinate, and $\mathbf{u}_{\phi}$ is the unit azimuthal vector, with $\phi$ the azimuthal angle. In 1997 Sadd, Chester, and Reatto [42] suggested that the velocity of superfluid ${ }^{4} \mathrm{He}$ is not truly singular at the vortex line and the vorticity is distributed over a finite region. Nevertheless, the deviations from Eq. (13) in ${ }^{4} \mathrm{He}$ seem to be very small [42].

We recall that there are remarkable differences between superfluid ${ }^{4} \mathrm{He}$ and the dilute superfluid we are considering here. In ${ }^{4} \mathrm{He}$ the effective radius $R_{0}$ of the interatomic potential is of the order of the average distance $n^{-1 / 3}$ between atoms, i.e., $R_{0} n^{1 / 3} \simeq 1$. Instead, in dilute ultracold gases the effective radius $R_{0}$ is always much smaller than the average distance $n^{-1 / 3}$ between atoms, i.e., $R_{0} n^{1 / 3} \ll 1$. For a dilute gas the coupling regime depends on the scattering length $a$, namely on the gas parameter $a n^{1 / 3}$ : in the weak-coupling regime $a n^{1 / 3} \ll 1$, while in the strong-coupling regime $a n^{1 / 3} \gg 1$. Another remarkable difference between liquid helium and quantum gases of alkali-metal atoms is the width of the vortex core. The core of a quantized vortex is only a few angstroms in superfluid ${ }^{4} \mathrm{He}$ while it is of the order of submicron in a dilute atomic gas [43].

In our USE we get Eq. (13) by setting $\boldsymbol{\Psi}(\mathbf{r}, t)$ $=\psi(\rho, z) \exp \left[i\left(L \phi-\frac{\mu_{0}}{\hbar} t\right)\right]$, where $\mu_{0}$ is the chemical potential of the inhomogeneous superfluid, fixed by the normalization. In this way, Eq. (5) with Eq. (10) becomes

$$
\left[-\frac{\hbar^{2}}{2 m}\left(\nabla_{\rho}^{2}-\frac{L^{2}}{\rho^{2}}+\frac{\partial^{2}}{\partial z^{2}}\right)+U(\rho, z)+\mu(n, a)\right] \psi=\mu_{0} \psi,
$$

where $\nabla_{\rho}^{2}=\frac{1}{\rho} \frac{\partial}{\partial \rho}\left(\rho \frac{\partial}{\partial \rho}\right)$ is the Laplacian operator in the radial direction and $\frac{\hbar^{2} L^{2}}{2 m \rho^{2}}$ is the centrifugal term which determines the size of the vortex core, that is of the order of the healing length $l_{h}=\hbar L / \sqrt{2 m \mu_{0}}$ [44]. This expression is obtained by equating the centrifugal term to the chemical potential $\mu_{0}$.

We introduce scaled variables by using the characteristic length $l_{c}$ of the system. In particular, we make the following transformations: $\bar{\rho}=\rho / l_{c}, \bar{z}=z / l_{c}, \bar{a}=a / l_{c}, \bar{U}=U\left(m l_{c}^{2}\right) / \hbar^{2}$, and $\bar{\mu}_{0}=\mu_{0}\left(m l_{c}^{2}\right) / \hbar^{2}$, and $\bar{\psi}=\psi l_{c}^{3 / 2}$. In this way Eq. (14) becomes

$$
\begin{aligned}
& {\left[-\frac{1}{2} \frac{\partial^{2}}{\partial \bar{\rho}^{2}}-\frac{1}{2 \bar{\rho}} \frac{\partial}{\partial \bar{\rho}}-\frac{1}{2} \frac{\partial^{2}}{\partial \bar{z}^{2}}+\frac{L^{2}}{2 \bar{\rho}^{2}}+\bar{U}(\bar{\rho}, \bar{z})+\bar{\psi}^{4 / 3} f\left(\bar{\psi}^{2 / 3} \bar{a}\right)\right] \bar{\psi}} \\
& \quad=\bar{\mu}_{0} \bar{\psi}
\end{aligned}
$$

where $\bar{\psi}(\bar{\rho}, \bar{z})$ is assumed to be real.

\section{VORTEX IN A UNIFORM BOSE GAS}

Let us first consider the case $U(\rho, z)=0$. We suppose that the Bose gas is asymptotically uniform, i.e.,

$$
\bar{\psi}(\bar{\rho}, \bar{z}) \rightarrow 1
$$

for $\bar{\rho}, \bar{z} \rightarrow \infty$. This condition fixes the characteristic length $l_{c}$ that must be $l_{c}=n_{\infty}^{-1 / 3}$ with $n_{\infty}$ the uniform density at infinity. 
In this case the chemical potential is fixed by the asymptotic condition, from which one finds

$$
\bar{\mu}_{0}=f(\bar{a}) .
$$

In addition, we can choose a wave function which depends only on $\bar{\rho}$, and Eq. (15) becomes

$$
\left[-\frac{1}{2} \frac{\partial^{2}}{\partial \bar{\rho}^{2}}-\frac{1}{2 \bar{\rho}} \frac{\partial}{\partial \bar{\rho}}+\frac{L^{2}}{2 \bar{\rho}^{2}}+\bar{\psi}^{4 / 3} f\left(\bar{\psi}^{2 / 3} \bar{a}\right)-f(\bar{a})\right] \bar{\psi}=0 .
$$

This second-order ordinary differential equation must be solved numerically. Clearly with $L \neq 0$ the vortex function $\bar{\psi}(\bar{\rho})$ has a core around $\bar{\rho}=0$. The asymptotic behavior of $\bar{\psi}(\bar{\rho})$ for $\bar{\rho} \rightarrow \infty$ is obtained by neglecting the spatial derivatives in Eq. (18). In this way we obtain the algebraic equation

$$
\bar{\psi}(\bar{\rho})^{4 / 3} f\left(\bar{\psi}(\bar{\rho})^{2 / 3} \bar{a}\right)=f(\bar{a})-\frac{L^{2}}{2 \bar{\rho}^{2}} .
$$

In the weak-coupling regime, $\bar{a} \ll 1$ where $f(x)=4 \pi x$, Eq. (19) gives

$$
\lim _{\bar{\rho} \rightarrow \infty} \bar{\psi}(\bar{\rho})=\left(1-\frac{L^{2}}{8 \pi \bar{a} \bar{\rho}^{2}}\right)^{1 / 2} .
$$

Instead, in the strong-coupling regime, $\bar{a} \gg 1$ where $f(x)=\xi$, Eq. (19) gives

$$
\lim _{\bar{\rho} \rightarrow \infty} \bar{\psi}(\bar{\rho})=\left(1-\frac{L^{2}}{2 \xi \bar{\rho}^{2}}\right)^{3 / 4} .
$$

Although the limit (20) depends on the scaled scattering length $\bar{a}$, the limit (21) is independent of $\bar{a}$. The scaled healing length $\bar{l}_{h}=l_{h} / l_{c}$, which estimates the size of the vortex core [44], is given by

$$
\bar{l}_{h}=L / \sqrt{2 f(\bar{a})} .
$$

Now we consider Eq. (18) in the limit $\bar{\rho} \rightarrow 0$. Because of the regularity of the wave function and the function $f(x)$ in this limit the last two terms [the terms involving the function $f(x)]$ in this equation remain finite and can be neglected in comparison to the angular momentum term $L^{2} /\left(2 \bar{\rho}^{2}\right)$. Consequently, we have the following condition at small $\bar{\rho}$ :

$$
\lim _{\bar{\rho} \rightarrow 0} \bar{\psi}(\bar{\rho})=\bar{\rho}^{L} \varphi(\bar{\rho})
$$

where $\varphi(\bar{\rho})$ is a smooth function.

We next solve Eq. (18) for $L=1$ and 2 using the classic fourth-order Runge-Kutta method [25,26]. This method produces very accurate convergence. We employ a space step of $\Delta=0.0001$ and integrate up to a scaled distance of $\bar{\rho}_{\max }=20$. The integration is started with the initial boundary condition (23) with a trial $\bar{\psi}(0)$ and $\bar{\psi}^{\prime}(0)$. For $L=1$ this condition is taken as $\bar{\psi}(0)=0$ and $\bar{\psi}^{\prime}(0)=$ const and the integration started at $\bar{\rho}=0$. For $L=2$ both $\bar{\psi}(0)=0$ and $\bar{\psi}^{\prime}(0)=0$ and the numerical integration cannot be started at $\bar{\rho}=0$, as then $\bar{\psi}$ as well as $\bar{\psi}^{\prime}$ at subsequent sites become zero. For $L=2$, the integration is started at $\bar{\rho}=\Delta$ with the boundary condition $\bar{\psi}(\Delta)=0$ and $\bar{\psi}^{\prime}(\Delta)$ equal to a small constant. The integration is propagated to $\bar{\rho}=\bar{\rho}_{\text {max }}$ where the asymptotic conditions (20) or (21) remain valid. If after integration with a trial guess, the boundary conditions (20) or (21) cannot be satisfied, the method is implemented with a new trial guess. The process is continued until a solution satisfying the proper boundary conditions at small and large $\bar{\rho}$ is obtained. We obtain the solution for different values of $\bar{a}$.

The numerical results for the scaled density $\left[\equiv \bar{\psi}^{2}(\bar{\rho})\right]$ for different scaled scattering length $\bar{a} \equiv a / l_{c}$ is plotted in Fig. 1 and compared with the asymptotic forms (20) or (21) for $L$ $=1$ and 2 . The corresponding parameters $\bar{\psi}^{\prime}(0)$ and $f(\bar{a})$ used in the numerical solution are given in Table I. The quantity $f(\bar{a})$ shown in Table I is interesting as it can be compared with the chemical potential of Eq. (18). Hence the numerical values of $f(\bar{a})$ for different $\bar{a}$ should give an idea of the variation of chemical potential with coupling. The quantity $f(\bar{a})$ is also related to the scaled healing length $\bar{l}_{h}$ given by Eq. (22) as shown in Table I.

It is interesting to calculate numerically the vortex core radius defined as the value of $\bar{\rho}$ for which $\bar{\psi}^{2}(\bar{\rho})=0.5$. In Fig. 2 we plot the numerical values of vortex core radius versus scaled scattering length and compare with the corresponding analytical results of healing length given by Eq. (22). The figure shows that the vortex-core radius has the same trend of the analytical healing length [44]. From Figs. 1 and 2 we find that the vortex-core radius decreases with increasing coupling but increases with angular momentum $L$. However, for very large coupling it saturates. This saturation of the vortex core is properly given by the USE.

\section{VORTEX IN A BOSE GAS IN A HARMONIC TRAP}

Now we consider the bosonic fluid in an axially symmetric harmonic trapping potential, i.e.,

$$
U=\frac{1}{2} m \omega_{\rho}^{2}\left(\rho^{2}+\lambda^{2} z^{2}\right),
$$

where $\lambda=\omega_{z} / \omega_{\rho}$ is the anisotropy parameter, with $\omega_{\rho}$ the transverse frequency and $\omega_{z}$ the axial frequency. We choose as characteristic length of the system the transverse harmonic length, i.e., $l_{c}=\sqrt{\hbar /\left(m \omega_{\rho}\right)}$. By using the scaled variables the confining potential reads $\bar{U}=\frac{1}{2}\left(\bar{\rho}^{2}+\lambda^{2} \bar{z}^{2}\right)$. With this confining potential the mean-field equation can be written explicitly as

$$
\begin{gathered}
{\left[-\frac{1}{2} \frac{\partial^{2}}{\partial \bar{\rho}^{2}}-\frac{1}{2 \bar{\rho}} \frac{\partial}{\partial \bar{\rho}}-\frac{1}{2} \frac{\partial^{2}}{\partial \bar{z}^{2}}+\frac{L^{2}}{2 \bar{\rho}^{2}}+\frac{1}{2}\left(\bar{\rho}^{2}+\lambda^{2} \bar{z}^{2}\right)\right.} \\
\left.+4 \pi N \bar{a} \bar{\psi}^{2} \frac{1+\alpha \bar{a}^{3 / 2} N^{1 / 2} \bar{\psi}}{1+\beta \bar{a}^{5 / 2} N^{5 / 6} \bar{\psi}^{5 / 3}}\right] \bar{\psi}=\bar{\mu}_{0} \bar{\psi}
\end{gathered}
$$

Now the normalization condition is given by 

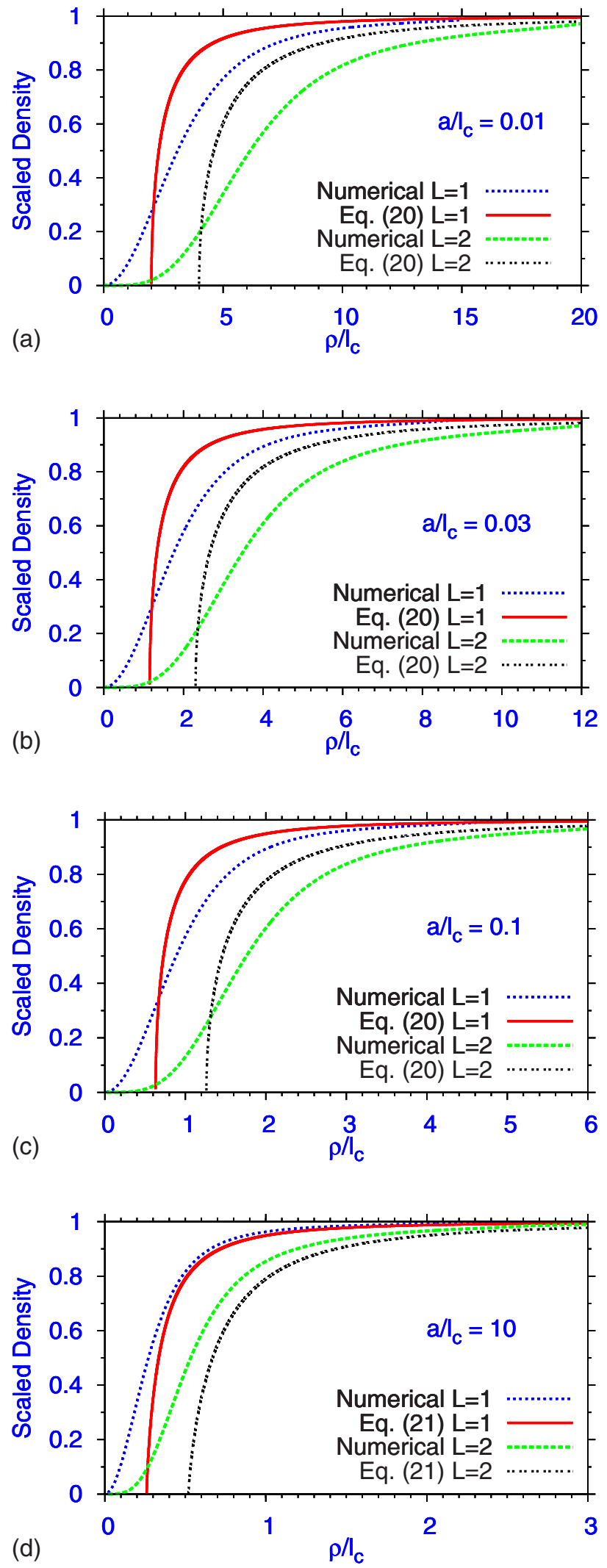

FIG. 1. (Color online) Numerical results for scaled density of vortices $\bar{\psi}^{2}(\bar{\rho})$ with $L=1$ and 2 in a uniform Bose gas obtained by solving the USE for scaled scattering lengths $a / l_{c}=0.01$ (a), 0.03 (b), 0.1 (c), and 10 (d) compared with the analytic asymptotic results (20) or (21).
TABLE I. The parameters for $L=1$ used in the numerical solution of Eq. (18) as plotted in Fig. 1; in this case $\bar{\psi}(0)=0$. For $L$ $=2, \bar{\psi}(0)=\bar{\psi}^{\prime}(0)=0$.

\begin{tabular}{llll}
\hline \hline $\bar{a}$ & $\bar{\psi}^{\prime}(0)$ & $f(\bar{a})$ & $\bar{l}_{h}=1 / \sqrt{2 f(\bar{a})}$ \\
\hline 0.01 & 0.2934232 & 0.126415651 & 1.989 \\
0.03 & 0.5157240 & 0.388573686 & 1.134 \\
0.1 & 1.0186620 & 1.47985617 & 0.581 \\
10 & 3.4622586006 & 22.3160243 & 0.149 \\
\hline \hline
\end{tabular}

$$
2 \pi \int_{0}^{\infty} \bar{\rho} d \bar{\rho} \int_{-\infty}^{\infty} d \bar{z} \bar{\psi}^{2}(\bar{\rho}, \bar{z}) \equiv 2 \pi \int_{0}^{\infty} \bar{\rho} d \bar{\rho} n_{c}(\bar{\rho})=1
$$

Here we defined $n_{c}(\bar{\rho})$ as the column density (as in [24]) appropriate for the study of radial distribution of matter in the vortex. Equations (25) and (26) constitute the USE for an axially trapped Bose gas valid from weak-coupling $(\bar{a} \longrightarrow 0)$ to the unitarity limit $(\bar{a} \rightarrow \infty)$ and (in spite of a slightly complicated numerical structure) it is no more difficult to solve than the usual GP equation valid in the weak-coupling limit. In the extreme weak-coupling limit, the nonlinear term of Eq. (25) becomes the usual GP term $4 \pi N \bar{a} \bar{\psi}^{2}$. In the unitarity limit this term becomes independent of scattering length and equals $\xi N^{2 / 3} \bar{\psi}^{4 / 3}, \xi=22.22$. We recall that if we use the GPE (7), as well as the MGPE (8), in the unitarity limit, it will lead to an inappropriate dependence of the nonlinearity on scattering length, as well as on the number of atoms $N$.

It is appropriate to study the behavior of the nonlinearity of the USE (25), that is $N^{2 / 3} \bar{\psi}^{4 / 3} f\left(N^{1 / 3} \bar{\psi}^{2 / 3} \bar{a}\right)$. To study the dependence of this nonlinearity on $N$ and $\bar{a}$ we set $\bar{\psi}=1$ in this expression. The resultant nonlinearities are plotted in Figs. 3(a) and 3(b) as a function of $\bar{a}$ and $N$ for constant $N$ $=1000$ and $a / l_{c}=0.05$, respectively. The interesting feature of the nonlinearity of this equation is exhibited in Fig. 3(a), where we find that, for a fixed $N$, the nonlinearity of this equation saturates at large scattering lengths $\bar{a}$. In the GP

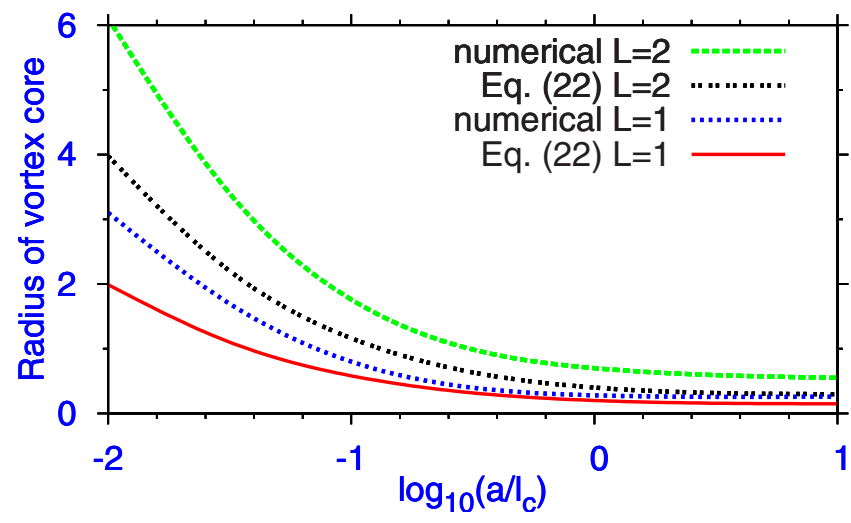

FIG. 2. (Color online) The numerical values of vortex core radius for $L=1$ and 2 vs scaled scattering length calculated by the USE compared with the analytic results of healing length given by Eq. (22). 

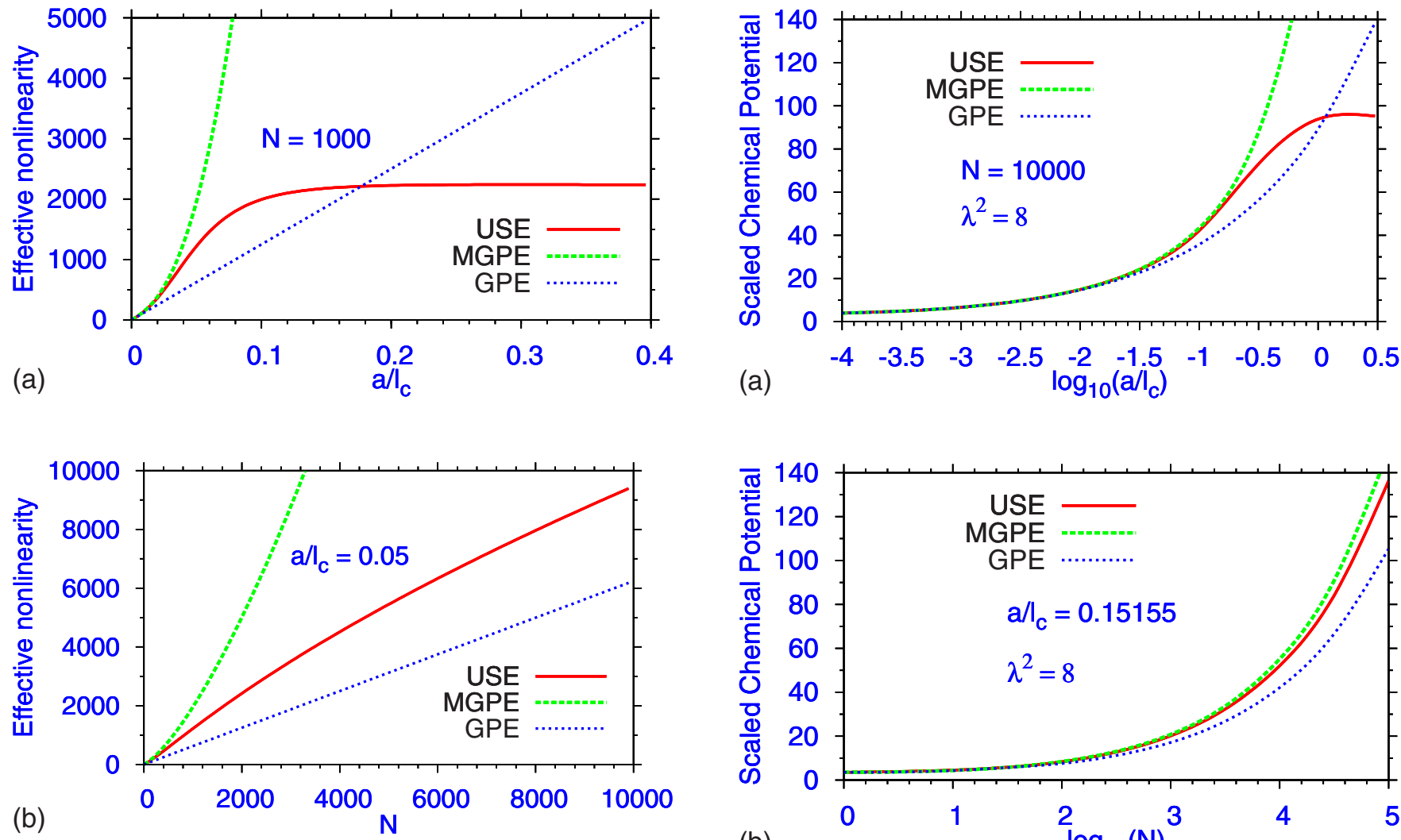

FIG. 3. (Color online) The numerical values of effective nonlinearity $N^{2 / 3} f\left(N^{1 / 3} \bar{a}\right)$ of the USE, $4 \pi N \bar{a}$ of the GPE, and $4 \pi N \bar{a}(1$ $+\alpha \bar{a}^{3 / 2} \sqrt{N}$ ) of the MGPE for $\bar{\psi}=1$ for (a) $N=1000 \mathrm{vs} a / l_{c}$ and (b) $a / l_{c}=0.05$ vs $N$.

model the nonlinearity increases linearly for all $\bar{a}$, whereas for the MGP model it increases indefinitely, but with a more complicated dependence on $\bar{a}$. From Fig. 3(b) we find that for a fixed scattering length the nonlinearity of Eq. (25) as well as the GP equation increases with $N$. For the GP equation it increases linearly with $N$, whereas for Eq. (25) it increases as $N^{2 / 3}$ for large $N$.

To further study the USE (25), we solve it numerically. For numerical convenience we transform this equation to its time-dependent form by replacing the term $\bar{\mu}_{0} \bar{\psi}$ by its timedependent counterpart $i \partial \bar{\psi} / \partial t$ appropriate for nonstationary states. However, in this paper we limit ourselves to a study of stationary states. Nevertheless, the introduction of timedependence allows for a solution of Eq. (25) by the imaginary time propagation method after discretizing it with the semi-implicit Crank-Nicholson rule [26-28]. In the process of discretization we use a time step of 0.0005 and a space step of 0.03 . We limit our numerical study to the $L=1$ vortex, as $L>1$ vortices are unstable and decay to several $L=1$ vortices conserving the angular momentum. In the numerical simulation of a vortex with $L=1$ we employ a pancakeshaped condensate with trap anisotropy $\lambda^{2}=8$ as in the theoretical study of Nilsen et al. [24] and the experiment at the Joint Institute for Laboratory Astrophysics, Colorado (JILA) [45]. After the wave function $\bar{\psi}(\bar{\rho})$ of Eq. (25) is obtained by the imaginary time propagation, the chemical potential is ob-

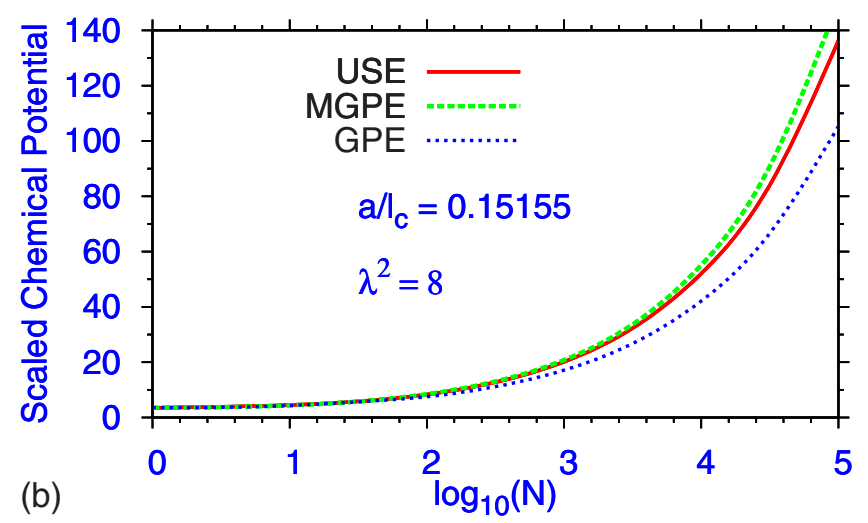

FIG. 4. (Color online) The numerically calculated scaled chemical potential $\bar{\mu}_{0}$ (in units of oscillator energy $\hbar \omega_{\rho}$ ) from GPE, MGPE, and USE vs (a) scaled scattering length $a / l_{c}$ for $N$ $=10000, \lambda^{2}=8$ and vs (b) $N$ for $a / l_{c}=0.15155, \lambda^{2}=8$.

tained by multiplying this equation by $\bar{\psi}(\bar{\rho})$ and integrating over all space.

In Fig. 4(a) we plot the numerical results of scaled chemical potential vs scaled scattering length $a / l_{c}$ as obtained from the USE (25) as well as the GPE (7) for $N=10000$. In Fig. 4(b) we plot the same vs $N$ for $a / l_{c}=0.15155$. The scaled scattering length $a / l_{c}=0.15155$ is the value studied by Nilsen et al. and is appropriate for Rb atoms in an experimental trap used at JILA for a scattering length $a=35 a(\mathrm{Rb})$, where $a(\mathrm{Rb})=100 a_{0}\left(a_{0}\right.$ is the Bohr radius) is the experimental atomic scattering length of Rb. In Table II we plot some of the results for chemical potential $\bar{\mu}_{0}$ for different $\bar{a}$ and $N$ values. The first row in this table agrees with the calculation of Nilsen et al. for the GP and the MGP models.

From Fig. 4(a) and Table II we find a saturation of the present mean-field result for large scattering length in the unitarity limit. This is consistent with the saturation of the nonlinearity of the USE (25) in this limit. After this saturation is obtained, any further increase in the scattering length at a fixed $N$ does not change the chemical potential $\bar{\mu}_{0}$. Figure 4 must be compared with Fig. 3. For a fixed scattering length, as $N$ is increased the nonlinearity of the USE (25) keeps on increasing. As a consequence the chemical potential also increases indefinitely as $N$. In Fig. 4(b) the USE chemical potential is always greater than the GP one. On the other hand, for a fixed $N$, as $\bar{a}$ is increased the nonlinearity of the 
TABLE II. Scaled chemical potential $\bar{\mu}_{0}$ of GPE, MGPE, and USE for different $\bar{a}=a / l_{c}$, $N$, and GP nonlinearity $\bar{a} N$.

\begin{tabular}{lllcrc}
\hline \hline $\bar{a}$ & $N$ & $\bar{a} N$ & $\mu_{\mathrm{GPE}}$ & $\mu_{\mathrm{MGPE}}$ & $\mu_{\mathrm{USE}}$ \\
\hline 0.15155 & 500 & 75.775 & 13.19 & 15.62 & 15.27 \\
0.01 & 10000 & 100 & 14.63 & 14.88 & 14.88 \\
0.01 & 100000 & 1000 & 35.73 & 36.74 & 36.70 \\
0.01 & 1000000 & 10000 & 89.25 & 93.16 & 92.99 \\
0.1 & 10000 & 1000 & 35.73 & 43.32 & 42.10 \\
1 & 10000 & 10000 & 89.25 & 196.13 & 93.83 \\
3 & 10000 & 30000 & 138.40 & 460 & 95.26 \\
\hline \hline
\end{tabular}

USE saturates above a certain value of $\bar{a}$. This has a consequence in the results: With the increase of scattering length, as the system tends to the unitarity limit, there is a saturation of the present chemical potential and a crossover, beyond which the USE chemical potential becomes smaller than the GP one. From Fig. 4 we find that for moderate to small $\bar{a}$ values the results for chemical potential of the MGPE (8) [4] and USE (25) remain very close to each other. From Table II we find that for $N=10^{6}$, and $\bar{a}=0.01$ (corresponding to a large GP nonlinearity of $N \bar{a}=10000$ ) the chemical potentials of the MGPE and the USE differ by less than $0.2 \%$ whereas the chemical potentials of these two models differ from that of the GP model by about 3\%. (We recall that $\bar{a}=0.01$ is the typical experimental value of this quantity in the experiment at JILA $[2,45]$.) The chemical potentials of MGPE and USE start to differ for large $\bar{a}$ values (larger than the experimental value but attainable by the Feshbach resonance technique [3]): the chemical potential of the USE model exhibits saturation, whereas the chemical potential of the MGP model increases very rapidly with increasing $\bar{a}$ values which has been made explicit in the data in the last two rows of Table II, where the results of the MGPE [4] and USE show the main differences.

In Fig. 5 we demonstrate a typical profile of the probability density of a vortex with $L=1$ as obtained from the USE for $N=10000$ and $\bar{a}=1$, corresponding to a large GP nonlinearity of $N \bar{a}=10000$. The density is manifestly zero for $\rho$ $=0$ for the vortex state. However, both for theoretical and

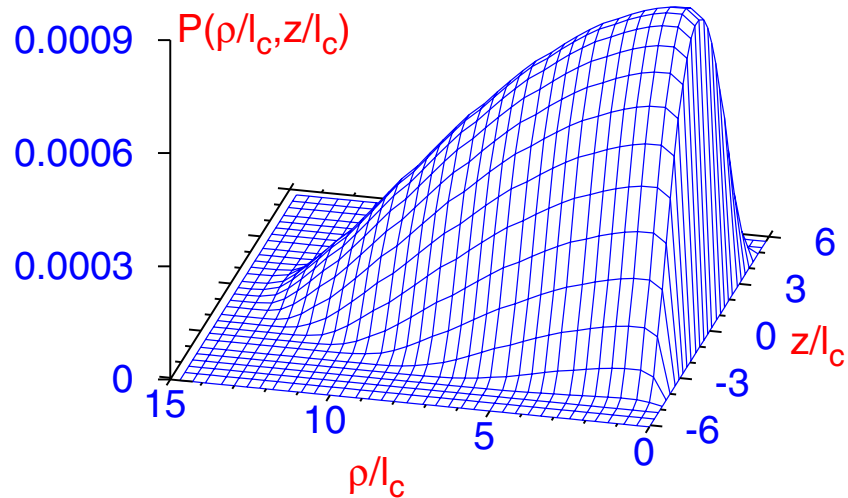

FIG. 5. (Color online) Typical profile of the probability density $P\left(\rho / l_{c}, z / l_{c}\right)=\bar{\psi}^{2}\left(\rho / l_{c}, z / l_{c}\right)$ for a vortex obtained with the USE for $N=10000$ and $\bar{a}=1$. experimental analysis it is appropriate and more convenient to calculate the column density $n_{c}(\bar{\rho})$ as defined in Eq. (26) to study the radial distribution of matter in a vortex for the USE, GPE, and MGPE. The results for column density are plotted in Fig. 6(a) for $N=10000$ for different $l / l_{c}$ and in Fig. $6\left(\right.$ b) for $a / l_{c}=0.15155$ for different $N$. The density profile of Fig. 6(b) for $N=500$ is also reported in [24] and the two agree with each other for the GP equation. An interesting feature of the plots of Fig. 6 is that with an increase of nonlinearity (either via an increase of $a / l_{c}$ for a fixed $N$, or via an increase of $N$ for a fixed $a / l_{c}$ ), the column density extends up to a larger radius. Of the column densities, that obtained with the USE extends to a larger radius than the GP
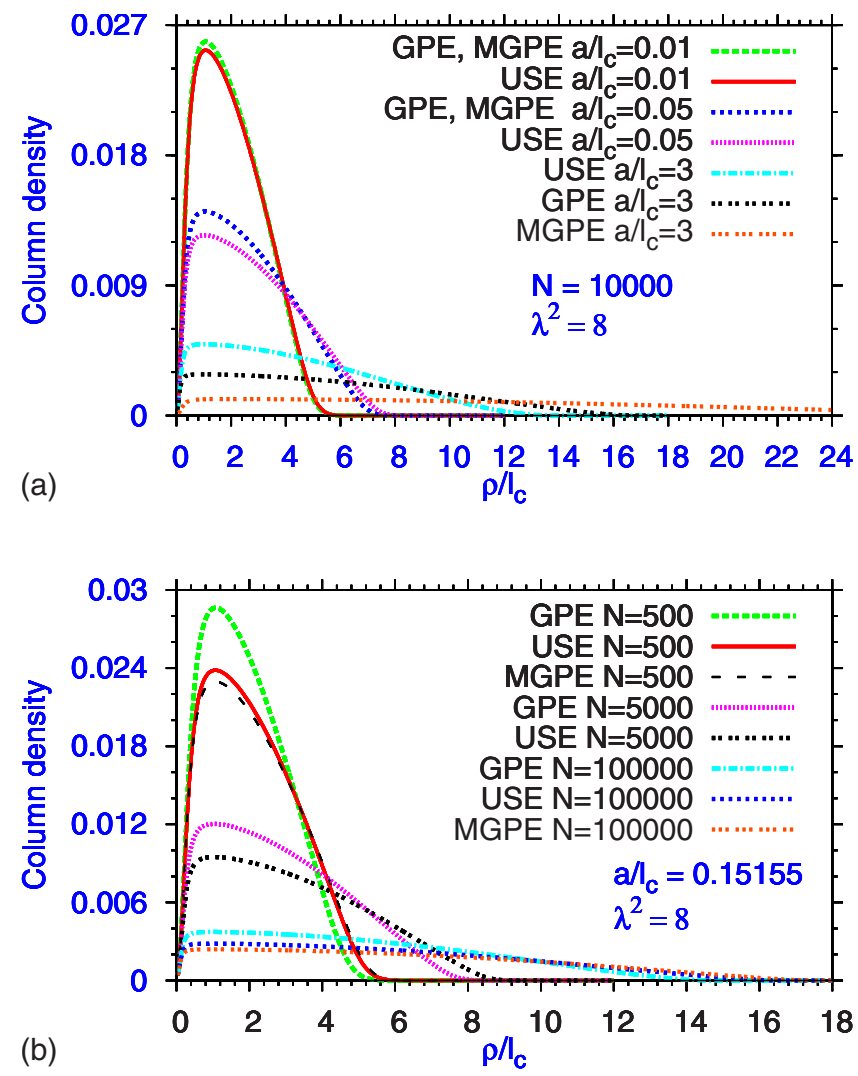

FIG. 6. (Color online) Numerically calculated column density $n_{c}(\bar{\rho})=\int_{-\infty}^{\infty} d \bar{z} \bar{\psi}^{2}(\bar{\rho}, \bar{z})$ from the USE, GPE, and MGPE vs $\rho / l_{c}$ for (a) $N=10000, \lambda^{2}=8$ and different $a / l_{c}$ and (b) $a / l_{c}=0.15155, \lambda^{2}=8$ and different $N$. 


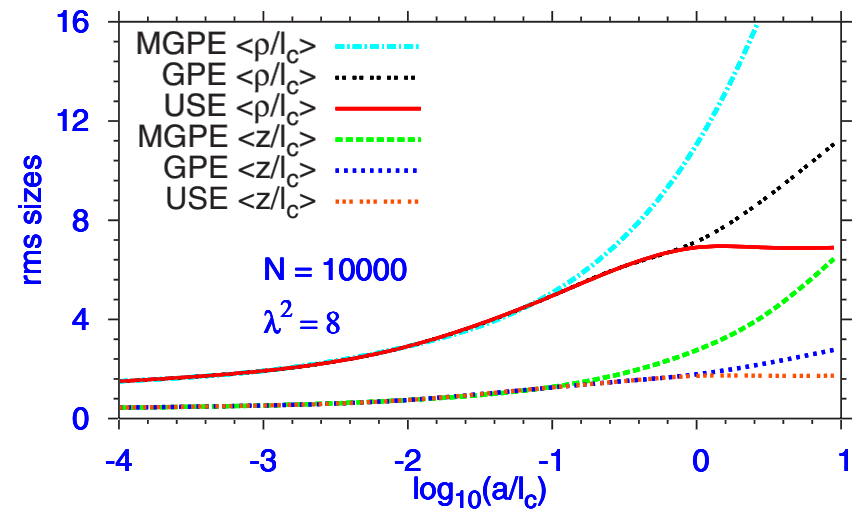

FIG. 7. (Color online) Numerically calculated scaled rms sizes $\left\langle\rho / l_{c}\right\rangle$ and $\left\langle z / l_{c}\right\rangle$ of the condensate from USE, GPE, and MGPE vs scaled scattering length $\bar{a}=a / l_{c}$.

column density in general. This trend is reversed in the unitarity limit of large scattering length as can be seen from the plot of Fig. 6(a) for $a / l_{c}=3$. For small values of $a / l_{c}$ the MGPE results are close to the GPE results. For medium values of $a / l_{c}$ the MGPE results are close to the USE results. However, for large values of $a / l_{c}$ the MGPE wave functions extend to very large distances, compared to the other models, due to an unphysically large nonlinearity in this model (MGPE).

A manifestation of the saturation of nonlinearity of the USE is explicit in the rms (root mean square) radial $\left(\left\langle\rho / l_{c}\right\rangle\right)$ and axial $\left(\left\langle z / l_{c}\right\rangle\right)$ sizes of the condensate as the scattering length $a$ is increased. The results for the rms sizes calculated with the GPE keeps on increasing indefinitely as the scattering length is increased. The rms sizes calculated with the MGPE increases even more rapidly than the results of the GPE. However, the rms sizes calculated with the USE saturates after a certain value of $a$, beyond which they remain constant. This is illustrated in Fig. 7 where we plot the rms sizes vs $\log \left(a / l_{c}\right)$ as calculated by the USE, GPE, and MGPE. As we are considering a pancake-shaped condensate the radial size $\left\langle\rho / l_{c}\right\rangle$ is larger than the axial size $z / l_{c}$. The theoretical results for the rms sizes exhibited in Fig. 7 can be verified experimentally with present technology and this will provide a test for the USE proposed in this paper.

\section{COLLECTIVE OSCILLATIONS IN HARMONIC CONFINEMENT}

We consider the effect of confinement due to an external anisotropic harmonic potential (24) on frequencies of collective oscillation of the system from weak-coupling to unitarity using the USE. It has been shown by Cozzini and Stringari [46] that assuming a power-law dependence $\mu=A n^{\Gamma}$ for the chemical potential (polytropic equation of state [47]), from Eqs. (1) and (2) without the quantum pressure term, one finds analytic expressions for the collective breathing frequencies of the superfluid. In particular, for the very elongated cigar-shaped traps, the collective radial breathing mode frequency $\Omega_{\rho}$ is given by [46]

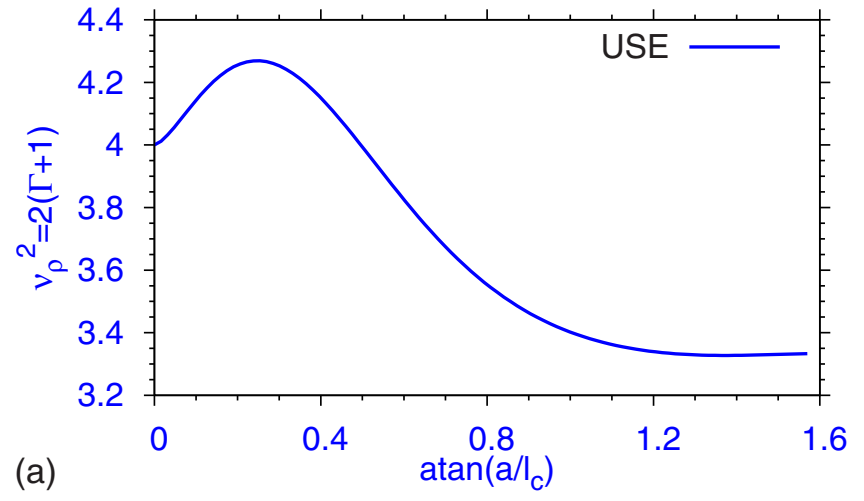

(b)

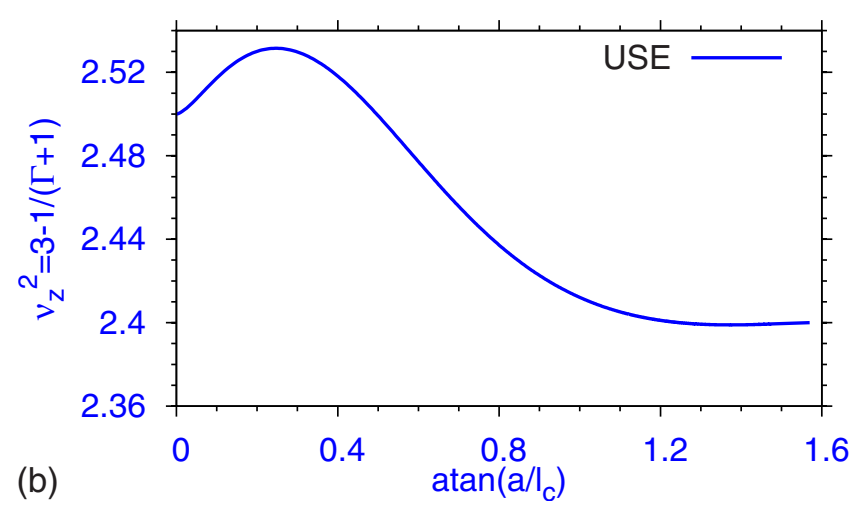

FIG. 8. (Color online) Square of the reduced frequencies of collective radial and axial oscillations (a) $\nu_{\rho}^{2}$ and (b) $\nu_{z}^{2}$ in a cigarshaped trap vs $\operatorname{atan}\left(a / l_{c}\right)$.

$$
\Omega_{\rho}=\sqrt{2(\Gamma+1)} \omega_{\rho},
$$

while the collective longitudinal breathing mode $\Omega_{z}$ is

$$
\Omega_{z}=\sqrt{\frac{3 \Gamma+2}{\Gamma+1}} \omega_{z} .
$$

Here we introduce an effective polytropic index $\Gamma$ as the logarithmic derivative of the bulk chemical potential $\mu$, that is

$$
\Gamma=\frac{n}{\mu} \frac{\partial \mu}{\partial n}=\frac{2}{3}+\frac{1}{3} x \frac{f^{\prime}(x)}{f(x)},
$$

where $x=n^{1 / 3} a$ is the gas parameter. This formula for the local polytropic equation is useful to have a simple analytical prediction of the collective frequencies. In the weakcoupling regime $(x \ll 1)$ one finds $x f^{\prime}(x) / f(x)=1$ and $\Gamma=1$, consequently, the reduced frequency square $\nu_{\rho}^{2} \equiv\left(\Omega_{\rho} / \omega_{\rho}\right)^{2}$ $=2(\Gamma+1)=4$ and $\nu_{z}^{2} \equiv\left(\Omega_{z} / \omega_{z}\right)^{2}=3-1 /(\Gamma+1)=5 / 2$; while in the unitarity regime $(x \gg 1)$ it holds $x f^{\prime}(x) / f(x)=0$ and $\Gamma$ $=2 / 3$ and consequently, $\nu_{\rho}^{2}=10 / 3$ and $\nu_{z}^{2}=12 / 5$. The analytical predictions of Eqs. (27) and (28) with Eq. (29) are shown in Fig. 8, where we plot $\nu_{\rho}^{2}$ and $\nu_{z}^{2}$ vs $\operatorname{atan}\left(a / l_{c}\right)$, so that the entire region $\infty>a \geq 0$ is mapped into the finite interval $\pi / 2 \geq \operatorname{atan}\left(a / l_{c}\right) \geq 0$. 


\section{CONCLUSION}

In this paper we have proposed a nonlinear Schrödinger equation-Eq. (12) or Eq. (25) — to study the properties of a BEC or a general superfluid Bose gas valid in both the weakcoupling and strong-coupling (or unitarity) limit. We call this equation the USE (unitary Schrödinger equation). This equation has a complicated nonlinearity structure in its dependence on scattering length $a$ and number of atoms $N$. In the extreme weak-coupling limit the USE (12) reduces to the usual mean-field GP Eq. (7) [2]; for medium coupling it becomes the modified GPE (MGPE) (8) introduced by Fabrocini and Polls [4], which is a generalization of the GP equation valid for medium coupling incorporating the correction to the bulk chemical potential of a Bose gas as introduced by Lee, Yang, and Huang [5]. However, for very strong coupling, both the GP and the MGP equations break down and the bulk chemical potential attains a saturation [8]. Considering this bulk chemical potential Cowell et al. [8] derived a nonlinear equation for a superfluid Bose gas valid in the strong coupling limit. The USE reduces to the equation by Cowell et al. in the strong coupling limit and thus has the correct form in both the weak and strong-coupling regimes. Hence this equation should be useful to study the crossover of a superfluid Bose gas from the weak- to strong-coupling limits. In the time-independent form the USE is useful to study the stationary properties of a BEC. The full timedependent USE can be used to study nonequilibrium properties of a dilute BEC, such as dynamical oscillations [28], collapse [48], free expansion after release from the trap [22], soliton formation and soliton dynamics [20,49], etc.

In this paper we applied the USE to study vortices in a superfluid Bose gas. First, we study vortices in a uniform Bose gas as the scattering length is varied from weak- to strong-coupling values. The vortex core radius decreases with increasing scattering length eventually attaining a saturation value. The vortex core radius is comparable to the healing length in this case.

Next, we study vortices in a BEC confined by an axially symmetric harmonic trap by solving the USE numerically. It is found that the effective nonlinearity of the USE saturates with the increase of scattering length $a$ in the strong-coupling limit for a fixed number of particles $N$. In this limit the chemical potential as well as the rms sizes of the BCS saturate attain constant values. However, the rms sizes obtained from the USE and the MGPE grows indefinitely as the scattering length is increased toward the unitarity limit. In the weak-coupling limit the results of the USE are compatible with those obtained from the GPE and the MGPE. Finally, we present results of axial and radial frequencies of collective oscillation of a BEC in a cigar-shaped trap using the USE.

The results of this paper for a BEC confined in an axiallysymmetric harmonic trap can be tested experimentally with present technology especially in the unitarity limit near a Feshbach resonance [3] and this will provide a stringent test for the proposed USE.

Recently, we became aware of some similar works [50].

\section{ACKNOWLEDGMENTS}

S.K.A. was partially supported by FAPESP and CNPq (Brazil), and the Institute for Mathematical Sciences of National University of Singapore. Research was (partially) completed while S.K.A. was visiting the Institute for Mathematical Sciences, National University of Singapore in 2007. L.S. was partially supported by GNFM-INdAM and Fondazione CARIPARO and thanks Francesco Ancilotto, Arturo Polls, Luciano Reatto, and Grigori Volovik for useful discussions.
[1] E. P. Gross, Nuovo Cimento 20, 454 (1961); L. P. Pitaevskii, Zh. Eksp. Teor. Fiz. 40, 646 (1961) [Sov. Phys. JETP 13, 451 (1961)].

[2] F. Dalfovo, S. Giorgini, L. P. Pitaevskii, and S. Stringari, Rev. Mod. Phys. 71, 463 (1999).

[3] S. L. Cornish, N. R. Claussen, J. L. Roberts, E. A. Cornell, and C. E. Wieman, Phys. Rev. Lett. 85, 1795 (2000); S. Inouye, M. R. Andrews, J. Stenger, H. J. Miesner, D. M. Stamper-Kurn, and W. Ketterle, Nature (London) 392, 151 (1998).

[4] A. Fabrocini and A. Polls, Phys. Rev. A 60, 2319 (1999); 64, 063610 (2001).

[5] T. D. Lee and C. N. Yang, Phys. Rev. 105, 1119 (1957); T. D. Lee, K. Huang, and C. N. Yang, ibid. 106, 1135 (1957).

[6] L. Pitaevskii and S. Stringari, Phys. Rev. Lett. 81, 4541 (1998).

[7] T. T. Wu, Phys. Rev. 115, 1390 (1959); E. Braaten and A. Nieto, Eur. Phys. J. B 11, 143 (1999).

[8] S. Cowell, H. Heiselberg, I. E. Mazets, J. Morales, V. R. Pandharipande, and C. J. Pethick, Phys. Rev. Lett. 88, 210403 (2002).

[9] H. Heiselberg, J. Phys. B 37, S141 (2004).
[10] J. F. Annett, Superconductivity, Superfluids and Condensates (Oxford University Press, Oxford, 2004); R. J. Donnelley, Quantized Vortices in Helium II (Cambridge University Press, Cambridge, England, 1991).

[11] C. Raman, J. R. Abo-Shaeer, J. M. Vogels, K. Xu, and W. Ketterle, Phys. Rev. Lett. 87, 210402 (2001).

[12] M. W. Zwierlein, J. R. Abo-Shaeer, A. Schirotzek, C. H. Schunck, and W. Ketterle, Nature (London) 435, 1047 (2005).

[13] F. Dalfovo and S. Stringari, Phys. Rev. A 53, 2477 (1996).

[14] A. A. Svidzinsky and A. L. Fetter, Phys. Rev. A 58, 3168 (1998).

[15] D. S. Rokhsar, Phys. Rev. Lett. 79, 2164 (1997).

[16] L. Salasnich, A. Parola, and L. Reatto, Phys. Rev. A 59, 2990 (1999).

[17] K. Kasamatsu, M. Tsubota, and M. Ueda, Phys. Rev. A 67, 033610 (2003); K. Kasamatsu, M. Machida, N. Sasa, and M. Tsubota, ibid. 71, 063616 (2005).

[18] S. K. Adhikari, Phys. Rev. E 65, 016703 (2001).

[19] L. Salasnich, Laser Phys. 14, 291 (2004).

[20] S. K. Adhikari, New J. Phys. 5, 137 (2003).

[21] S. K. Adhikari, Phys. Rev. A 66, 043601 (2002); 69, 063613 
(2004).

[22] S. K. Adhikari, Phys. Rev. A 65, 033616 (2002).

[23] S. K. Adhikari and L. Salasnich, Phys. Rev. A 75, 053603 (2007).

[24] J. K. Nilsen, J. Mur-Petit, M. Guilleumas, M. Hjorth-Jensen, and A. Polls, Phys. Rev. A 71, 053610 (2005).

[25] S. K. Adhikari, Phys. Lett. A 265, 91 (2000); Phys. Rev. E 62, 2937 (2000).

[26] S. E. Koonin and D. C. Meredith, Computational Physics Fortran Version (Addison-Wesley, Reading, 1990).

[27] E. Cerboneschi, R. Mannella, E. Arimondo, and L. Salasnich, Phys. Lett. A 249, 495 (1998); L. Salasnich, A. Parola, and L. Reatto, Phys. Rev. A 64, 023601 (2001).

[28] S. K. Adhikari and P. Muruganandam, J. Phys. B 35, 2831 (2002).

[29] L. Pitaevskii and S. Stringari, Bose-Einstein Condensation (Oxford University Press, Oxford, 2003).

[30] G. E. Volovik, in Proceedings of The Eleventh Marcel Grossmann Meeting on General Relativity, edited by H. Kleinert, R. T. Jantzen, and R. Ruffini (World Scientific, Singapore, 2008).

[31] L. D. Landau and E. M. Lifshitz, Fluid Mechanics, Course of Theoretical Physics (Pergamon Press, London, 1987), Vol. 6.

[32] L. D. Landau and E. M. Lifshitz, Statistical Physics, Part 2: Theory of the Condensed State, Course of Theoretical Physics (Pergamon Press, London, 1987), Vol. 9.

[33] W. Lenz, Z. Phys. 56, 778 (1929).

[34] H. Heiselberg, Phys. Rev. A 63, 043606 (2001); S. K. Adhikari, ibid. 76, 053609 (2007).

[35] E. Lipparini, Modern Many-Particle Physics: Atomic Gases, Quantum Dots and Quantum Fluids (World Scientific, Singapore, 2004).

[36] L. Salasnich, S. K. Adhikari, and F. Toigo, Phys. Rev. A 75, 023616 (2007); S. K. Adhikari and L. Salasnich, ibid. 76, 023612 (2007).
[37] N. Manini and L. Salasnich, Phys. Rev. A 71, 033625 (2005); G. Diana, N. Manini, and L. Salasnich, ibid. 73, 065601 (2006); L. Salasnich and N. Manini, Laser Phys. 17, 169 (2007).

[38] L. Salasnich, A. Parola, and L. Reatto, Phys. Rev. A 70, 013606 (2004); 72, 025602 (2005).

[39] G. V. Chester, R. Metz, and L. Reatto, Phys. Rev. 175, 275 (1968).

[40] F. Dalfovo, Phys. Rev. B 46, 5482 (1992).

[41] S. Stringari and J. Treiner, Phys. Rev. B 36, 8369 (1987).

[42] M. Sadd, G. V. Chester, and L. Reatto, Phys. Rev. Lett. 79, 2490 (1997).

[43] M. Tsubota, J. Phys.: Conf. Ser., 31, 88 (2006)

[44] K. W. Madison, F. Chevy, W. Wohlleben, and J. Dalibard, Phys. Rev. Lett. 84, 806 (2000); N. G. Berloff and P. H. Roberts, Phys. Lett. A 274, 69 (2000); K. Staliunas, Appl. Phys. B: Lasers Opt. 71, 555 (2000).

[45] M. H. Anderson, J. R. Ensher, M. R. Matthews, C. E. Wieman, and E. A. Cornell, Science 269, 198 (1995).

[46] M. Cozzini and S. Stringari, Phys. Rev. Lett. 91, 070401 (2003).

[47] R. Combescot and X. Leyronas, Phys. Rev. Lett. 93, 138901 (2004).

[48] E. A. Donley, N. R. Claussen, S. L. Cornish, J. L. Roberts, E. A. Cornell, and C. E. Wieman, Nature (London) 412, 295 (2001); S. K. Adhikari, Phys. Rev. A 66, 013611 (2002); C. M. Savage, N. P. Robins, and J. J. Hope, ibid. 67, 014304 (2003); W. Bao, D. Jaksch, and P. A. Markowich, J. Phys. B 37, 329 (2004).

[49] L. Khaykovich et al., Science 296, 1290 (2002); K. E. Strecker et al., Nature (London) 417, 150 (2002).

[50] A. Banerjee and M. P. Singh, Phys. Rev. A 66, 043609 (2002); 73, 033607 (2006); G. S. Nunes, J. Phys. B 32, 4293 (1999). 